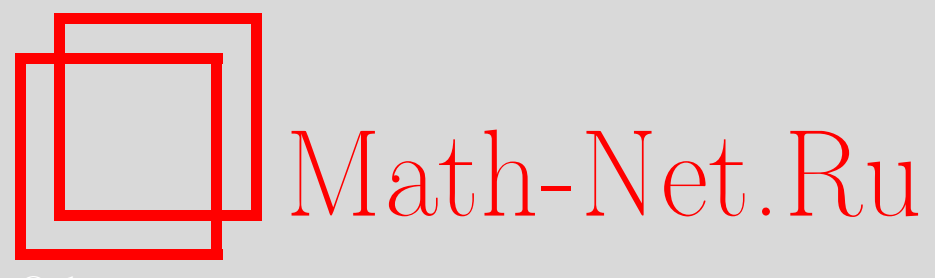

Ф. Григорьев, Период ангармонических колебаний, Квант, 2019, номер 8, 42-43

DOI: https://doi.org/10.4213/kvant20190805

Использование Общероссийского математического портала Math-Net.Ru подразумевает, что вы прочитали и согласны с пользовательским соглашением http://www.mathnet.ru/rus/agreement

Параметры загрузки:

IP : 54.166 .219 .16

26 апреля 2023 г., $13: 36: 14$

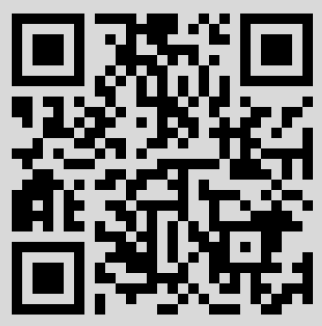




\section{Период \\ ангармонических колебаний}

Ф.ГРИГОРЬЕВ

\begin{abstract}
АРМОНИЧЕСКИМИНАЗЫВАЮТ КОЛЕБА-
ния, при которых ускорение тела после смещения из положения равновесия направлено к положению равновесия и прямо пропорционально величине смещения. Период гармонических колебаний не зависит от амплитуды и максимальной скорости колебаний.
\end{abstract}

В реальных колебательных системах закон прямой пропорциональности ускорения и смещения часто нарушается с ростом амплитуды колебаний. Например, колебания математического маятника становятся $a \boldsymbol{u}^{-}$ гармоническими, если амплитуда колебаний сравнима с длиной нити или если угол отклонения нити от вертикали перестает быть малым по сравнению с единицей (величина угла выражается в радианах).

Если слагаемые в возвращающей силе, пропорциональные более высокой степени смещения, чем первая, много меньше линейного слагаемого, то ангармонизм колебаний может быть рассмотрен как малое возмущение, дающее поправку в зависимость смещения от времени и в период колебаний. Однако в некоторых случаях ангармонизм не может быть учтен таким образом. Рассмот-

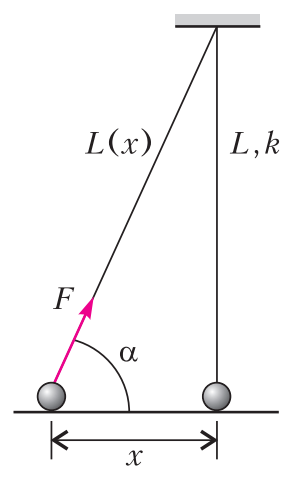

рим пример такой системы.

Пусть гладкий шарик массой $m$ лежит на горизонтальной поверхности и прикреплен к нижнему концу подвешенной недеформированной легкой резинки жесткостью $k$ и длиной $L$ (см. рисунок). Если отклонить шарик от положения равновесия на

DOI: https://doi.org/10.4213/kvant20190805 малую величину $x \ll L$ и отпустить, то под действием силы упругости резинки $F$, горизонтальная составляющая которой направлена против смещения, возникнут колебания:

$$
m a_{x}=-F \cos \alpha=-\frac{k \Delta L}{L} x,
$$

где $\Delta L=L(x)-L-$ деформация резинки. На первый взгляд, в уравнении (1) ускорение прямо пропорционально смещению, как и в гармоническом законе, однако это не так. Дело в том, что деформация тоже зависит от смещения. Чтобы сделать эту зависимость более удобной для рассмотрения, преобразуем $\Delta L$ с учетом условия малости колебаний $x \ll L$ :

$$
\begin{aligned}
\Delta L=L(x)-L=\frac{L^{2}(x)-L^{2}}{L(x)+L} & \\
& \approx \frac{\left(L^{2}+x^{2}\right)-L^{2}}{L(x)+L} \approx \frac{x^{2}}{2 L} .
\end{aligned}
$$

Подставив это выражение в уравнение (1), получим

$$
m a_{x}=-\gamma x^{3},
$$

где $\gamma=k /\left(2 L^{2}\right)$. Мы видим, что ускорение пропорционально кубу смещения. Такие колебания не будут гармоническими, так как зависимость вида $x(t)=A \cos \left(\omega t+\varphi_{0}\right)$ не является решением уравнения (2) в произвольный момент времени, в чем можно убедиться непосредственной подстановкой.

Интересно, что период таких колебаний, в отличие от периода гармонических колебаний, зависит от амплитуды колебаний $A$. Покажем это, исходя из соображений размерности. Период $T$ может зависеть только от $\gamma, m$ и $A$. Поскольку $\gamma / m$ имеет размерность $\mathrm{M}^{-2} \cdot \mathrm{c}^{-2}$, есть только одна комбинация этих величин, имеющая размерность времени:

$$
T \sim\left(\frac{1}{A}\right) \cdot\left(\frac{m}{\gamma}\right)^{1 / 2},
$$

откуда следует, что период колебаний описанной системы обратно пропорционален амплитуде.

Точно найти период можно, опираясь на закон сохранения механической энергии $E$ при колебаниях с потенциальной энергией 
$U(x)$ :

$$
\frac{m v_{x}^{2}}{2}+U(x)=E
$$

Выразим отсюда скорость, рассматривая движение на участке, на котором проекция скорости положительна:

$$
\frac{d x}{d t}=\left(\frac{2(E-U(x))}{m}\right)^{1 / 2} .
$$

Разделим переменные:

$$
d t=\frac{d x}{(2(E-U(x)) / m)^{1 / 2}} .
$$

Шарик проходит расстояние, равное амплитуде колебаний, за четверть периода. Возьмем интеграл от обеих частей последнего равенства:

$$
\frac{T}{4}=\int_{0}^{A} \frac{d x}{\sqrt{2(E-U(x)) / m}} .
$$

Чтобы вычислить правую часть, необходимо знать зависимость потенциальной энергии упругой резинки от смещения. Учитывая связь проекции силы и потенциальной энергии: $F_{x}=-d U / d x$, а также равенство нулю потенциальной энергии в положении равновесия, найдем

$$
U(x)=\frac{\gamma x^{4}}{4} .
$$

Поскольку в крайних положениях скорость равна нулю, механическая энергия колебаний выражается через амплитуду следующим образом: $E=\gamma A^{4} / 4$. Используя замену переменных $x=z A$, для периода колебаний получим

$$
T=\left(\frac{4}{A}\right) \cdot\left(\frac{2 m}{\gamma}\right)^{1 / 2} \int_{0}^{1} \frac{d z}{\sqrt{1-z^{4}}} .
$$

Входящий в это выражение интеграл не зависит от характеристик колебательной системы. Он выражается через специальные функции (бета-функции) и приблизительно равен 1,3. Впрочем, его можно найти численно на компьютере. Учитывая, что $\gamma=k /\left(2 L^{2}\right)$, период можно выразить через жесткость и длину резинки:

$$
T \approx\left(\frac{10 L}{A}\right) \cdot\left(\frac{m}{k}\right)^{1 / 2} .
$$

Интересно сравнить период ангармонических колебаний с периодом гармонических колебаний, возникающих, если в начальном состоянии резинка растянута на $\Delta L$, а амплитуда колебаний $A \ll \Delta L$. Проекция второго закона Ньютона на направление смещения в этом случае имеет вид

$$
m a_{x}=-\frac{k \Delta L}{L} x
$$

и период колебаний равен

$$
T=2 \pi\left(\frac{m L}{k \Delta L}\right)^{1 / 2} .
$$

Теперь найдем отношение периодов ангармонических и гармонических колебаний:

$$
\frac{T_{\text {анг }}}{T_{\text {г }}}=\left(\frac{5}{\pi}\right) \cdot\left(\frac{L \Delta L}{A^{2}}\right)^{1 / 2} .
$$

Это отношение не зависит от жесткости резинки и массы шарика. Заметим также, что при условии примерного равенства амплитуд гармонических и ангармонических колебаний период последних будет существенно больше.

Зависимость периода колебания от амплитуды - типичная особенность ангармонических колебаний. Например, если возвращающая сила пропорциональна пятой степени смещения:

$$
m a_{x}=-\beta x^{5},
$$

то период колебаний обратно пропорционален квадрату амплитуды:

$$
T=\left(\frac{4}{A^{2}}\right) \cdot\left(\frac{3 m}{\beta}\right)^{1 / 2} \int_{0}^{1} \frac{d z}{\sqrt{1-z^{6}}},
$$

где интеграл приблизительно равен 1,2. 